termed the Hispanic paradox. Recent research suggests that this phenomenon may be partly explained by the areas in which Hispanic people live. Hispanic mothers living in counties with a high proportion of Hispanic people (Hispanic density) have lower rates of infant mortality and smoking during pregnancy. In this paper, we investigate whether or not Hispanic density is associated with better birth outcomes for mothers of other ethnicities.

Design Multilevel analysis of the US Linked Birth and Infant Death Dataset 2000 and US census data at county level.

Setting USA.

Participants 2274247 White and 581151 Black non-Hispanic mothers of singleton births.

Main outcome measures Infant mortality, low birthweight, preterm delivery and maternal smoking during pregnancy.

Results Living in counties with a higher percentage of Hispanic residents was associated with reduced risk of all outcomes for nonHispanic White and Black mothers and infants in analyses adjusting for individual and area level socio-demographic characteristics. The reduction in odds was greatest for maternal smoking during pregnancy. White mothers living in counties where more than half the residents were Hispanic had their odds of smoking during pregnancy reduced by approximately $80 \%$ (OR $0.1995 \%$ CI 0.11 to 0.33 ), relative to comparable mothers living at Hispanic densities of 0 to $1 \%$. Similar reductions in risk of maternal smoking during pregnancy were found for Black mothers (OR 0.14 $95 \%$ CI 0.14 to 0.51 ). Infant mortality was reduced by approximately a third for both Black and White mothers living in counties with a high proportion of Hispanic residents. In addition, higher Hispanic density was associated with modest but significant reductions in the risks of preterm delivery and low birthweight.

Conclusions Living in Hispanic communities appears to have health benefits for those of non-Hispanic origin.

\section{COMPARISON OF DISTRIBUTIONS OF COMMON INDICATORS OF SOCIO-ECONOMIC POSITION BY ETHNICITY AND MIGRATION: PRELIMINARY FINDINGS FROM THE BORN IN BRADFORD BIRTH COHORT STUDY}

doi:10.1136/jech.2010.120956.55

${ }^{1} \mathrm{~L}$ Fairley, ${ }^{2} \mathrm{~N}$ Small, ${ }^{3} \mathrm{D}$ A Lawlor, ${ }^{1} \mathrm{~J}$ Wright. ${ }^{1}$ Bradford Institute for Health Research, Bradford Royal Infirmary, Bradford, UK; ${ }^{2}$ School of Health Studies, University of Bradford, Bradford, UK; ${ }^{3}$ MRC Centre for Causal Analyses in Translational Epidemiology, University of Bristol, Bristol, UK

Objective To describe and compare the distributions, including extent of missing data, in measures of socio-economic position (SEP) between women of white British and Pakistani ethnicity. We also compare distributions of measures of SEP between Pakistani women born in UK and those born in Pakistan and also compare distributions by the woman's age at migration to the UK.

Design The Born in Bradford birth cohort study recruits pregnant women at 26-28 weeks gestation when they complete a baseline questionnaire; approximately half these women are of Pakistani origin.

Setting Bradford, UK.

Participants Data are currently available for 2005 White British and 2444 Pakistani women.

Main outcome measure Indicators of SEP included in these analyses are: the woman's education and employment, her partner's education and employment and household income.

Results $57 \%$ of the Pakistani ethnicity women were born in Pakistan and there was a bimodal distribution of age at migration to the UK with peaks at ages 1 and 18 years. 92\% of the White British women were, or had been, in paid employment compared to $51 \%$ of the
Pakistani women. This figure was $82 \%$ for UK-born Pakistani women, $73 \%$ for those born in Pakistan who moved aged 5 or under and $22 \%$ for those who moved after the age of 5 . Overall $23 \%$ of women reported that they didn't know their family income; this varied by ethnicity and country of birth (8\% for White British women, 21\% for UK-born Pakistani women and 49\% for those born in Pakistan). The percentage of women reporting an income of less than $£ 20000$ was highest in Pakistani women, while the percentage of women reporting an income of $£ 20000$ and over was highest for the White British women. 11\% of White British women had no educational qualifications compared to $22 \%$ of Pakistani women; this figure was lowest for UK-born Pakistani women. The percentage of women with degree level education was higher in Pakistani than White British women (26\% and 19\%, respectively) and was similar for all Pakistani women irrespective of migration history. The percentage of women's partners with no qualifications was similar between ethnic groups, however the percentage of partners with degree level education was higher for the Pakistani women.

Conclusion These differences in the distributions of SEP measures by ethnicity and migration are important to understand health inequalities and for ensuring appropriate adjustment of SEP confounding.

\section{SOCIO-DEMOGRAPHIC, HEALTH STATUS, PSYCHO-SOCIAL AND LIFESTYLE PREDICTORS OF SELF-RATED HEALTH IN THE ALL IRELAND TRAVELLER HEALTH STUDY}

doi:10.1136/jech.2010.120956.56

${ }^{1} \mathrm{~J}$ Whelan, ${ }^{1} \mathrm{P}$ Fitzpatrick, ${ }^{1} \mathrm{~S}$ Abdalla, ${ }^{1} \mathrm{~F}$ Cronin, ${ }^{1} \mathrm{~A}$ Drummond, ${ }^{1} \mathrm{~L}$ Daly, ${ }^{1} \mathrm{~K}$ Frazier, ${ }^{1} \mathrm{~N}$ A Hamid, ${ }^{1} \mathrm{C}$ Kelly, ${ }^{1} \mathrm{C}$ McGorrian, ${ }^{1} \mathrm{R}$ G Moore, ${ }^{1} \mathrm{R}$ NicCharthaigh, ${ }^{1} \mathrm{~B}$ O'Shea, ${ }^{1} \mathrm{D}$ O'Mahony, ${ }^{1} \mathrm{~B}$ Quirke, ${ }^{2} \mathrm{~A}$ Staines, ${ }^{1} \mathrm{D}$ Staines, ${ }^{2} \mathrm{M} \mathrm{R}$ Sweeney, ${ }^{1} \mathrm{~J}$ Turner, ${ }^{1} \mathrm{C} C$ Kelleher. ${ }^{1}$ UCD School of Public Health, Physiotherapy and Population Science, University College Dublin, Belfield, Dublin, Republic of Ireland; ${ }^{2}$ School of Nursing, Dublin City University, Dublin, Republic of Ireland

Background Irish Travellers have a lower life expectancy than the general Population. Objective health-status indices are consistently poorer in Traveller and Gypsy populations than in other comparably deprived social groups. Self-rated Health (SRH) is established as a valid indicator of objective health status.

Methods As part of an all-Ireland census of 10618 Traveller families both North and South (response rate $80 \%$ overall) employing a novel audio-visual computer interview methodology with peer researchers to overcome literacy barriers, a personal, structured interview was conducted with a random $20 \%$ sub-sample of adults $(n=2065,43.5 \%$ male). This analysis describes predictors of SRH in those adults, within health domains such as socio-demographic, environmental, lifestyle and psychosocial factors (including those related to culture/ identity, and to social capital such as experiences of discrimination). Six models were constructed initially for each domain separately and then a final backwards stepwise logistic regression model was chosen that included 36 potential predictors from all domains of health, with self rated health as a outcome variable dichotomised into categories good (excellent, very good or good) vs fair or poor.

Results SRH was rated as excellent, good or very good by $82.6 \%$ of respondents and was age-related. Those who reported limiting disability/long-term illness were excluded. Each separate domain model showed variables predictive of SRH. On adjustment for agegroup and sex in the social capital model for instance, experience of discrimination (OR 0.5, $\mathrm{p}=0.02$ ), low levels of trust (OR 0.6, $p=0.04$ ) and worry about getting fair treatment (OR 0.6, $p=0.007$ ) were independently inversely associated with good SRH. In the final model, independent positive predictors of good SRH were having a flush toilet ( $\mathrm{OR} 2.2, \mathrm{p}=0.02$ ), considering where you live to be 
healthy (OR 1.9, $\mathrm{p}=0.02)$, going "on the road" at least twice in the last year (OR 2.3, $\mathrm{p}=0.03$ ) and a brisk walk at least once in the last week (OR 2.4, $\mathrm{p}=0.000)$. Good SRH was inversely associated with increasing age-group $(p=0.000)$, smoking ( $O R \quad 0.6, p=0.03)$, activitylimiting ill health for $\geq 1$ day in the last month (OR $0.4, p=0.001$ ), chronic health condition diagnosed by the GP (OR 0.4, $\mathrm{p}=0.002$ ), and having been screened for hypertension, high cholesterol or diabetes (OR 0.6, $\mathrm{p}=0.03$ ).

Conclusion There is a clear relationship between established health determinants and SRH in the Traveller community, suggesting the importance of both material and psychosocial factors. The directionality of the relationship between cultural factors and lifestyle cannot be inferred in these models.

\section{ALL IRELAND TRAVELLER HEALTH STUDY: INCREASING GAP IN MORTALITY BETWEEN TRAVELLER AND GENERAL POPULATIONS IN THE REPUBLIC OF IRELAND OVER TWO DECADES}

doi:10.1136/jech.2010.120956.57

S Abdalla, B Quirke, L Daly, P Fitzpatrick, C Kelleher. UCD School of Public Health, Physiotherapy and Population Science, Dublin, Republic of Ireland

Background Irish Travellers are a distinct minority group characterised by a nomadic lifestyle, specific culture and substantial socioeconomic and health disadvantage. When examined in 1987 the Traveller community in the Republic of Ireland (ROI) were shown to have a higher mortality than the general population. Updated information was needed to inform policy action in this area.

Objective To examine the current mortality experience of Irish Travellers in the ROI and to contrast it with that of the general population.

Design, setting and participants We conducted a retrospective mortality count as part of a wider Traveller Census (The All Ireland Traveller Health Study). In ROI 9056 Traveller families were surveyed. Census respondents were asked to identify all immediate and extended family members who died over an exact 12 month period preceding the census date. Information obtained was corroborated with and supplemented by reports from the Traveller health networks, Public Health Nurses and General Registrar death records.

Main outcome measures Standardised mortality ratios (SMR) with 95\% CI using the 2008 ROI general population mortality rates as standard.

Results There were 188 Traveller deaths in the year preceding the census date of October $14^{\text {th }}$ 2008. Traveller mortality in ROI is nearly three and a half times higher than that of the general population (SMR 348; 95\% CI 298 to 397). This compares with a corresponding SMR of 254 calculated in 1987. Though general population mortality (age-adjusted) has declined by $35 \%$ over the past 20 years, Traveller mortality has only dropped by $13 \%$, thus widening the mortality gap. Males have a significantly higher mortality than females with an SMR of 469 (95\% CI 387 to 552) compared to a female SMR of 232 (95\% CI 175 to 289). With a standard set of general population mortality rates in those aged 15 years and over, Travellers had an SMR of 277 (95\% CI 235 to 319) compared to an SMR of 232 (95\% CI 227 to 237 ) in the lower socioeconomic group of the general population.

Conclusion Mortality among Irish Travellers has declined over the past 20 years but at a slower rate than that of the general population; the gap between Travellers and the general population has widened, particularly in males. The current gap is larger than that between the lower socio-economic group and the general ROI population. The results highlight the value of mortality measurement in uncovering health trends and health inequalities.

\section{Pregnancy and/or smoking \\ 058 JUST LOOK FOR AN ASHTRAY: PRAGMATIC FACTORS ASSOCIATED WITH SMOKING RELAPSE POSTPARTUM}

doi:10.1136/jech.2010.120956.58

${ }^{1} \mathrm{~S}$ Prady, ${ }^{2} \mathrm{~K}$ Keirnan, ${ }^{1} \mathrm{~K}$ Bloor, ${ }^{1} \mathrm{~K}$ Pickett. ${ }^{1}$ Department of Health Sciences, University of York, UK; ${ }^{2}$ Department of Social Policy and Social Work, University of York, UK

Objective Most women who stop smoking during their pregnancy will relapse within the first 6 months after birth, with health risks to the mother and family. There have been few UK-specific studies identifying factors associated with relapse. Such information would help health care providers identify those most at risk so that extra support and resources can be targeted. We examined whether a small set of factors that are potentially readily measured or observable in routine health care settings were associated with women at risk for relapse postpartum.

Design and setting Analysis of the first wave of the Millennium Cohort Study. Variables in the final model were selected using backwards logistic regression weighted to account for the complex survey design.

Participants 2353 natural mothers who reported quitting smoking during their pregnancy.

Main outcome measure Postpartum smoking relapse.

Results $58 \%$ of mothers who quit during pregnancy were smoking again at 9 months. Only $16.2 \%$ of smoking couples quit together. The strongest risk factors were related to postnatal partner smoking status and single parenthood. Compared to mothers with a nonsmoking partner, the risk of relapse was higher for those with a partner who smoked at 9 months (OR 3.2, 95\% CI 2.6 to 3.9), a partner who smoked antenatally $(1.2,1.4$ to 2.1$)$, and those who had no live-in partner postnatally $(3.2,2.2$ to 4.8$)$ or antenatally $(2.3,1.5$ to 3.4). Other significant risk factors remaining in the models were the mother having no-one to share her feelings with, drinking 1-2 times a month or 1-2 times a week, having fewer educational qualifications and more children in the household. Factors that did not appear to be influential in the final models were age, ethnicity, change in marital status from birth to 9 months, depression history, financial status, timing of entry into ante-natal care, feelings about the pregnancy or breastfeeding.

Conclusion Pragmatic variables can be used to identify women at higher risk for relapse. The validity of these findings are potentially limited by the inability to measure antenatal intent to relapse, the smoking cessation and relapse prevention interventions actually received, and timing/sequence of relapse for partner-mother pairs in this data set. Both partner smoking habits and single marital status are strongly associated with relapse and can be readily identified both antenatally and postnatally by healthcare providers

\section{MATERNAL AND PATERNAL SMOKING DURING PREGNANCY AND TRAJECTORIES OF GROWTH AND ADIPOSITY IN THE OFFSPRING}

doi:10.1136/jech.2010.120956.59

${ }^{1,2} \mathrm{~L}$ D Howe, ${ }^{3} \mathrm{~A}$ Matijasevich, ${ }^{1} \mathrm{~K}$ Tilling, ${ }^{1,2}{ }^{\mathrm{D}} \mathrm{A}$ Lawlor. ${ }^{1}$ Department of Social Medicine, University of Bristol, Bristol, UK; ${ }^{2}$ MRC Centre for Causal Analyses in Translational Epidemiology, University of Bristol, Bristol, UK; ${ }^{3}$ Post-graduate Programme in Epidemiology, Federal University of Pelotas, Pelotas, Brazil

Objective To explore the associations of maternal smoking during pregnancy with offspring trajectories of height, weight and adiposity and to compare these with associations with paternal smoking to determine whether any associations are driven by intrauterine mechanisms. 